\title{
The Design of User-centric Mobile Crowdsensing with Cooperative D2D Communications
}

\author{
Shusen Yang, Xiaofei Wang, Usman Adeel, Cong Zhao, Jia Hu, Xinyu Yang, and Julie McCann
}

\begin{abstract}
The ubiquitous sensor-rich smartphones have promoted Mobile Crowdsensing (MCS), an emerging people-centric sensing paradigm for urban Internet-ofThings (IoTs). However, using cellular networks to transmit the big data collected by MCS would incur expensive financial costs for both participating phone users and the MCS organizer. A promising solution to this is to integrate the low-cost Devices-to-Device (D2D) communication into the MCS design. Yet using D2D in MCS will require the cooperative interactions among the self-interest and strategic participating phone users, which significantly complicates the "human-in-the-loop" MCS design in both digital and human dimensions, and brings a branch of new challenges: (1) data communication and networking become more complex; (2) D2D connections among opportunistically encountered phone users must be secure, fast, and user transparent; and (3)participating phone users need to be properly incentivized. In dealing with these challenges, this article will cover recent developments of D2D-enabled MCS from both theoretical and practical perspectives. Future research questions in this rapidly growing field are also discussed.
\end{abstract}

Index Terms-Mobile Crowdsourcing, Human-In-TheLoop, Secure Device Pairing, Incentivization

\section{INTRODUCTION}

The ubiquitous sensor-rich smartphones have been playing an increasing important role in the evolution of urban Internet of Things (IoTs), bridging the digital space and the physical world at a citywide scale. This promotes the emergence of a fast-growing sensing paradigm, the Mobile CrowdSensing (MCS) [1], [2]. The sheer numbers of mobile users combined with the increasing powerful computing/communication capabilities of modern phones, make MCS a much more flexible and cost-effective sensing paradigm than traditional

Shusen Yang with the National Engineering Laboratory for Big Data Analytics (NEL-BDA) at Xi'an Jiaotong University.

Xiaofei Wang is with the Tianjin Key Laboratory of Advanced Networking (TANK) at Tianjin University.

Usman Adeel is with the Intel Labs Europe.

Cong Zhao and Julie McCann are with the Department of Computing at Imperial College London.

Jia Hu is with the College of Engineering at University of Exeter.

Xinyu Yang (Corresponding Author) is with the Department of Computer Science and Technology at Xi'an Jiaotong University. sensor networks. Furthermore, the inherent mobility of phone users enables increased sensing coverage both spatially and over time, providing opportunities to collect data at a higher granularity and with more penetration.

A variety of big data reported by numerous mobile users produce rich knowledge (e.g. human mobility, social context, user feelings/opinions, traffic, and air qualities), which enables a broad range of data-driven applications and services such as urban planing, public safety, transportation and environment monitoring [1][3]. However, the huge volume of data collected by MCS incur expensive financial costs (i.e. phone bills) [4]-[8], which significantly restricts the scale and popularity of MCS applications. Recent work shows that applying the cheap (or free) Devices-to-Device (D2D) communications [9] (e.g. WiFi direct and Bluetooth smart) in the MCS design is promising to solve this issue, especially in the dense urban crowd scenarios and MCS applications with limited financial budget.

However, besides the direct and reliable interactions between each individual MCS participator and the server in the traditional cellular-only MCS systems, applying the opportunistic D2D communications will also require the additional cooperative interactions among the selfinterest and strategic mobile users. This will significantly complicates the "human-in-the-loop" design [10] of MCS in both digital and human dimensions, and brings a branch of new research challenges. Especially, the following three issues are specific and essential for the success of D2D-enabled MCS:

1) Networking Algorithms to achieve adaptiveness to the potentially stochastic and unpredictable D2D communication opportunities, while maximizing the performance gain of utilizing D2D communications.

2) Device Pairing Schemes to simultaneously ensure secure, trustworthy, fast, and user-transparent D2D connections between opportunistically encountered phone users, who are normally strangers with strong security and privacy concerns.

3) Incentivization Mechanisms to guarantee the faithful cooperative behaviors of the self-interest and strategic phone users. 


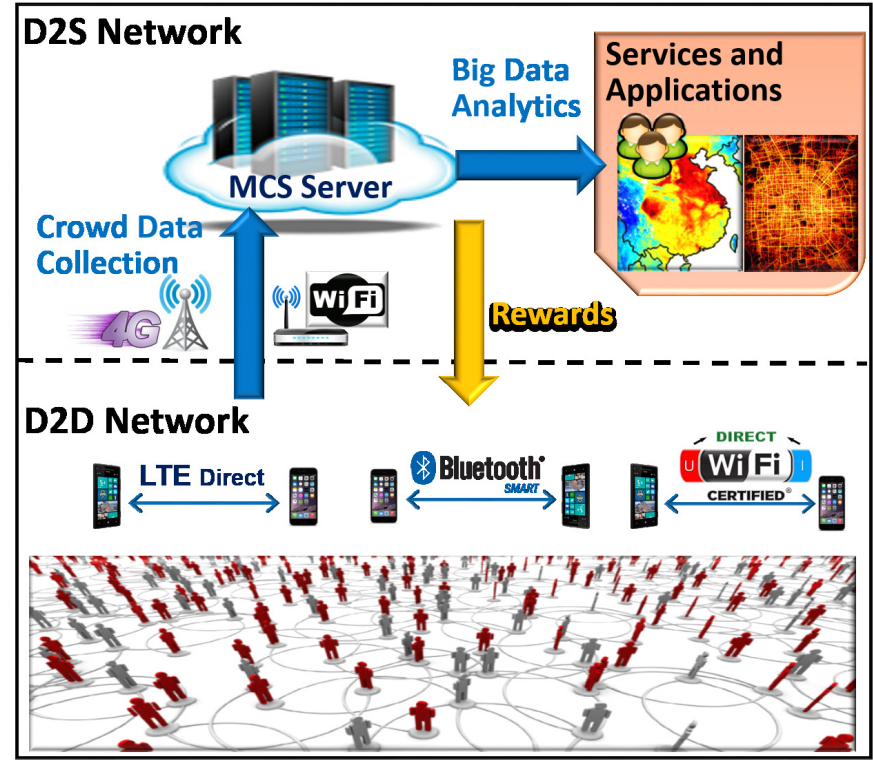

Fig. 1. Illustration of a general framework for MCS-D2D.

In this article, we will provide an overview of D2Denabled MCS by discussing these challenges and stateof-the-art solutions from both theoretical and practical perspectives and in a systematic way. We will also discuss several important research challenges that lie ahead.

\section{A General Framework of MCS with COOPERATIVE D2D COMMUNICATIONS}

\section{A. General Framework}

A general framework of a MCS with cooperative D2D communications is illustrated in Fig.1. A typical MCS system consists of a server and a set of participating mobile phones that continuously collect sensory data and report the collected data to the server. The collected data will feed various data-driven applications and services. However, fulfilling the MCS tasks will incur costs in both phone bills and phone resources (CPU, memory, battery, and network bandwidth). Therefore, proper monetary reward should be paid to the mobile users to motivate their participation [4], [5], [7], [8], [11].

A straightforward way to upload the collected data is to use the cellular network, which is adopted in most existing MCS designs [1], [2]. Alternatively, a mobile user can also report his or her sensory data to the server via other phones through a sequence of opportunistic short-range D2D transmissions in a "carry-and-forward" fashion. D2D communication techniques (e.g. the WiFi direct, Bluetooth, and LTE direct) are considered as an important component in the 5G communication system, which can support various applications such as content sharing, gaming, wireless relaying, and cellular offloading. A recent complete and comprehensive survey for D2D communication techniques and applications can be found in [9].

For instance, without using its own cellular radio, a phone user can report his or her sensory data through another proximity phone with a cheaper cellular cost (e.g. unlimited mobile data) or when he or she passes a free WiFi router. As shown in Fig. 1, such D2D-enabled MCS system have two subnetworks for data transmission: a Device-to-Server (D2S) network with always-connected cellular links and opportunistic WiFi links, and a mobile multi-hop D2D network with time-varying D2D links between opportunistically encountered phone users.

\section{B. Advantages of Applying D2D Communications in MCS Design}

Recently, there have been more and more practical systems that demonstrate the great potential of opportunistic D2D communications. For instance, Fig.2 illustrates one-week D2D transmission activities of a mobile APP Xender $^{1}$, including over 20 million transmissions for more than one million mobile users in India. Although Xender is not an MCS APP, and only a small part of its traces are demonstrated, the D2D transmission capacity shown in Fig.2 demonstrate the great potential of D2D-enabled MCS for the large-scale data collections. Specifically, in comparison with pure cellular communications, the opportunistic D2D communication brings the following benefits to the MCS systems:

- Throughput Improvement. Considering the high density of urban crowd, sophisticated short-range radios in smartphones, and the increasing population of public WiFi routers; the opportunistic D2D network has a great potential data delivery capacity for practical urban MCSs. This significantly improves the throughput of the MCS network to support the big sensory data collection. Although the throughput gain is achieved at the expense of sacrificing transmission latency, many MCS applications do not require strict real-time data communications, such as environmental monitoring, human mobility, and social contexts [1]-[3].

- Financial Cost Reduction. The phone bill paid for mobile data usage is the major financial cost for MCS, especially for high sampling rate, multimedia (e.g. images and videos), and continuous sensing applications. The great expense incurred to the MCS organizer for phone bills would severely restrict the

${ }^{1}$ http://www.xender.com/. 


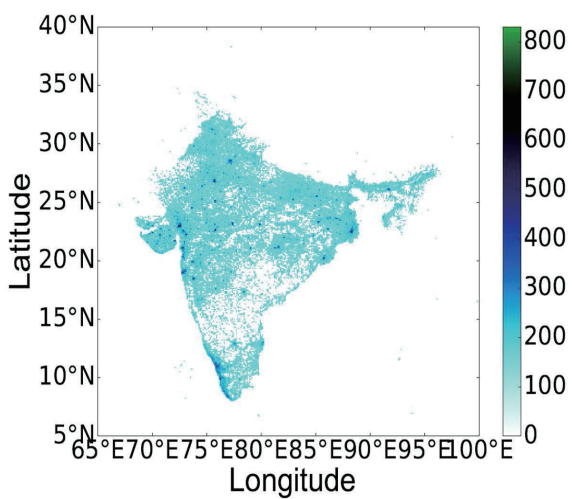

(a)
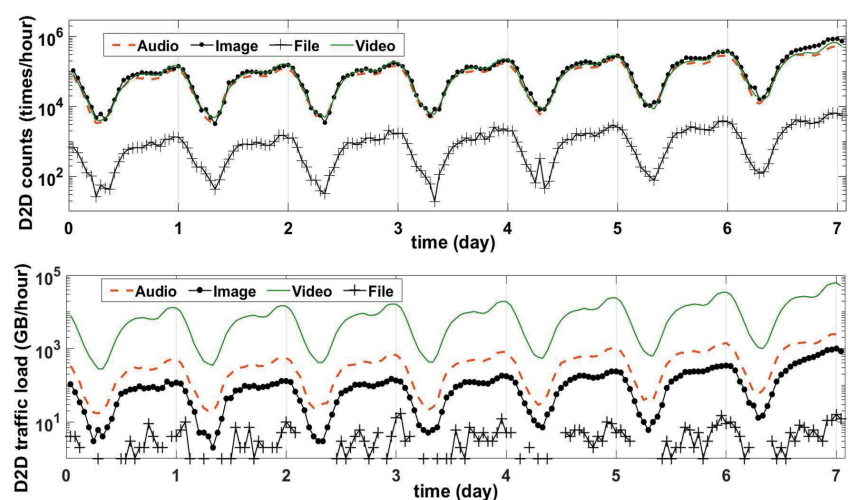

(b)

Fig. 2. Real-world D2D transmission activities of an mobile APP Xender: a) Heatmap of Xender users' activities; b) D2D transmissions between Xender users over a week, which demonstrates the great data offloading capacities of opportunistic D2D communications.

performance practical MCS applications. In contrast, a D2D-enabled MCS can achieve significant cellular traffic offloading and use almost-free opportunistic short-range communications, which has a great potential to collect big crowd data with a much smaller budget.

- Prolonging Battery Lifetime. Typical short-range D2D communication radios consume less power than the long-range cellular communications. This results in longer battery lifetime and therefore less degradation of mobile user experience, further benefiting user participation.

\section{Key Design Challenges}

Despite the benefits discussed above, D2D communications require the cooperative interactions of opportunistically-encountered participating phone users, which significantly complicate the "human-in-the-loop" MCS design in both digital and human dimensions, as shown in Fig. 3. Specifically, the following three essential issues must be addressed to ensure the success of D2D-enabled MCS.

1) Networking. Different from cellular-based MCSs, new networking algorithms should be developed for D2D-enabled MCSs, which can adapt to the time-varying and potentially unpredictable network states; such as the fluctuating wireless channel quality, intermittent connectivity caused by phone user movement, and the opportunistic availability of free WiFi networks. Specifically, networking algorithms are expected to achieve the four Essential Properties (EPs) summarized in Fig. 3.

2) Mobile Device Pairing. As shown in Fig. 3, the D2D connections between mobile devices require fast, user-transparent, and secure device paring. This is important not only in supporting the upperlayer networking algorithms, but also in enabling the trustworthy interactions among MCS participants, which therefore covers both the digital and human design spaces.

3) Incentivization. To address the incentivization issues caused by the social and economics behaviors of the participating "human sensor", a number of mechanisms (typically auction-based approaches) have been proposed for traditional cellular-only MCS [12], which only requires the interactions between each individual phone user and MCS server. Besides such individual interactions, D2Denabled MCS would require the participating users to perform the sensing and data communication tasks in a cooperative way, which introduce a new dimension of research issues that do not exist in cellular-only MCS. For instance, a participating phone may maliciously drop the sensor data received from other phones through D2D communications, in order to reduce its own memory and battery cost. Such unfaithful behaviors would result in severe performance degradation, and therefore must be inherently prevented by design proper incentivization mechanisms.

Above three issues are highly related and will be discussed in detail with state-of-the-art results in later sections.

\section{DyNAMIC NETWORKING FOR HYBRID D2S AND D2D NETWORKS}

In order to achieve D2D-enabled MCS, the first challenge is to design efficient networking algorithms to support sensor data delivery over the hybrid D2S and 


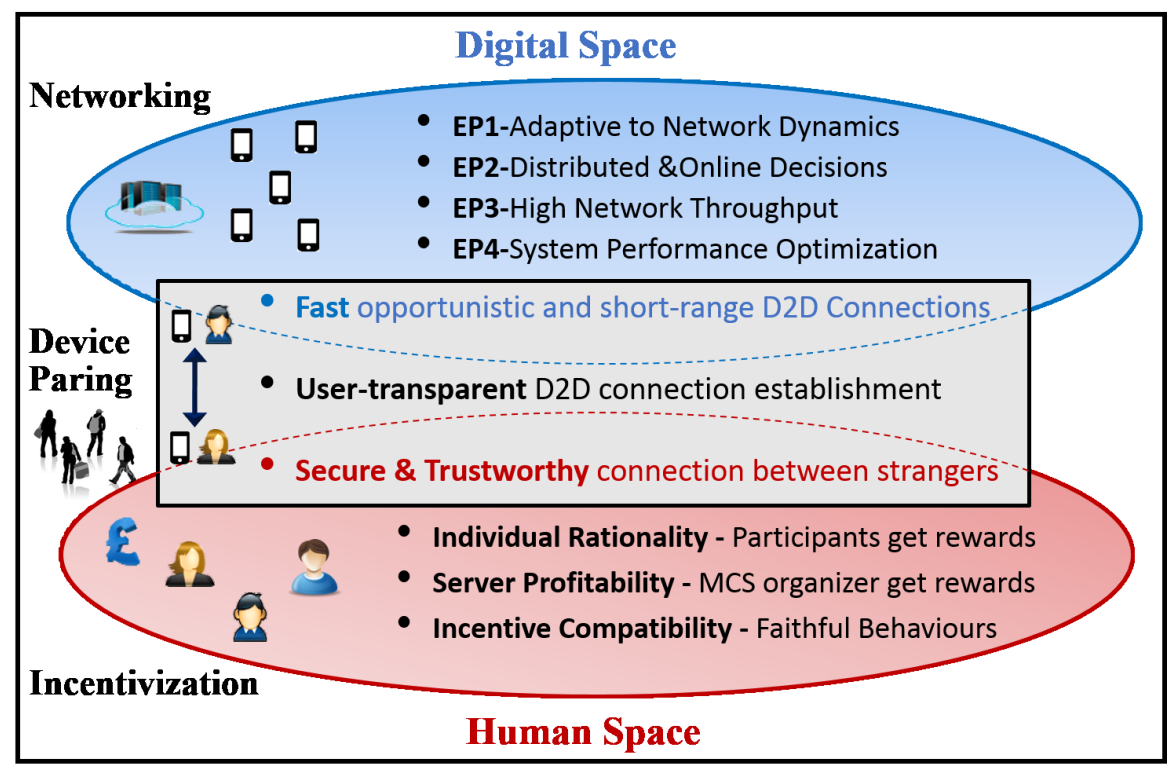

Fig. 3. The relations of the three key technique issues of D2D-enabled MCS.

D2D networks shown in Fig.1. Specifically, from the perspective of each participating phone $x$, the following " $4 \mathrm{~W}$ " questions regarding data communications need to be answered:

- When should $x$ use cellular networks and when opportunistic short-range communications?

- Who send data to $x$ and who receive data from $x$ ?

- Which D2D neighbor and radio (e.g. WiFi Direct or Bluetooth) should be selected for data forwarding?

- What are the data rates at which $x$ collects, forwards, and receives sensory data respectively?

\section{A. Four Essential Properties of Networking Algorithms in D2D-enable MCS}

To answer above "4W" questions, a networking algorithm to support D2D-enabled MCS is expected to achieve the following four Essential Properties (EPs).

- EP1-Adaptive to Complex Network Dynamics. The states of a D2D-enabled MCS is highly dynamic and stochastic, depending on various random timevarying factors (e.g. wireless interference and channel qualities, sensing tasks arrivals, and human mobility), governed by various complex physical rules. An expected algorithm should be adaptive to all these possible dynamic network states. For instance, the expected algorithm should work well from the extremely dense network, where all phones can always communicate with each other, to the extremely sparse case where no phone can communicate with others through D2D links.

- EP2-Distributed and Online Decision Making. Considering the citywide scale of MCSs, distributed algorithms are preferred due to scalability concerns and robustness to network failures. In addition, the network algorithm should be lightweight and agile enough for online decision makings, to adapt to network dynamics.

- EP3-High Throughput for Data Delivery. The potential network capacity gain achieved by D2D networks should be sufficiently exploited to support the huge volume of MCS data.

- EP4-System Performance Optimization. The expected algorithm should aim at optimizing the performance of the D2D-enabled MCS system, such as minimization of overall system monetary costs, maximization of global system profit, maximization of cellular traffic offloading, or multi-objective optimization of these performance metrics.

\section{B. A Case Study: Lynaponov Network Optimization for D2D-Enabled MCSs}

Recent work [7], [8], [11] has demonstrated that backpressure algorithms driven by Lynaponov Optimization Theory ${ }^{2}$ is a promising family of networking approaches to achieve EPs 1-4 for the D2D-enabled MCS design. A general mathematical formulation of stochastic optimization for D2D-enabled MCS is as follows:

Minimize $\operatorname{cost}(\overline{\mathbf{x}}, \mathbf{S})-\operatorname{reward}(\overline{\mathbf{x}}, \mathbf{S})$

Subject to Equality constraints

Inequality constraints

All queues are bounded

${ }^{2}$ https://en.wikipedia.org/wiki/Lyapunov_optimization 
where $\overline{\mathbf{x}}$ represents long-term averages of control actions $\mathbf{x}(t)$ for all time $t$ (e.g. sensing and data transmission decisions of all phones); $\mathbf{S}$ represents all stochastic system states (e.g. mobile users connectivity and D2D channel quality). The objective is to find the best control actions $\mathbf{x}(t)$ to minimize a cost function (or maximize a reward function such as social profits), by satisfying equality and inequality constraints (e.g. channel capacity constraint), and ensuring bounded data queues for all phones. A family of adaptive algorithms, backpressure algorithms driven by Lyapunov optimization theory [7], [8], [11], are throughput optimal (EP3), make control decision at real-time (EP1), and have strong theoretical performance guarantees (EP4). Furthermore, if the objective and constraint functions are separable, then lightweight and fully distributed network algorithms can be developed (EP2).

However, a natural question now is whether these theoretical algorithms work in practice? To answer this question, we constructed an experimental WiFiDirect-enabled MCS consisting of 10 Android phones (held by volunteering students from Imperial College London) and a MCS server implemented in NODE.JS (http://nodejs.org/), as shown in Fig. 4. We implemented a fully distributed and real-time backpressure based routing algorithm to minimize the overall system costs:

- Data Structure. Each phone $x$ maintains its current sensing rate $r_{x}(t)$, its queue backlog $Q_{x}(t)$, a system parameter $V>0$, and a temporal neighbor table to record the information of its all one-hop neighbors $\mathcal{N}_{x}(t)$, which includes the server and all other phones can communicate with $x$ trough WiFi direct links. For instance, phone $x$ in Fig. 4 has three neighbors $\mathcal{N}_{x}(t)=\{\mathrm{A}, \mathrm{B}, \mathrm{S}\}$ at time $t$.

- Routing and Data Forwarding. At each time slot $t$, each phone $x$ makes the following online decisions: (1) Routing: $x$ computes a weight $w_{x, y}(t)=$ $Q_{x}(t)-Q_{y}(t)-V \operatorname{cost}_{x, y}(t)$ for each of its temporal neighbor $y \in \mathcal{N}_{x}(t)$, where $\operatorname{cost}_{x, y}(t)$ is the current cost of data forwarding over link $(x, y)$, depending on link types and qualities (Fig. 4 shows an example). Then $x$ finds the best neighbor $y^{*}$ with the maximal weight (i.e. $w_{x, y^{*}} \geq w_{x, y}, \forall y \in \mathcal{N}_{x}(t)$ ). (2) Data Forwarding: If the maximal weight is positive (i.e. $w_{x, y^{*}}(t)>0$ ), $x$ forwards data to $y^{*}$.

This simple algorithm has strong theoretical performance guarantees. Specifically, it can be proven that the minimal system costs of the D2D-enabled MCS system can be asymptotically achieved, as the system parameter $V \rightarrow+\infty$ [7].

The experiment lasted for three days, during which each phone run the backpressure routing algorithm and were never turned off. During the experiment, nearly all D2D communications happened within the Imperial College Campus on working times, because there were only 10 phones and their owners live in different places across London. We set the parameter $V=100$ and sensing rate as 2 samples (data packets) per minute ${ }^{3}$. The transmission cost for each cellular communication link was randomly set between 1 and 10, while for each WiFi and WiFi direct link was set as 1 . As shown in Fig 4, above simple backpressure algorithm achieves $1,4520 / 4,5150 \approx 32.2 \%$ cellular traffic offloading. In addition, the average end-to-end delay is around half hour, which is acceptable by many MCS applications such as environmental monitoring. These results were exciting given the sparse network settings, and it is reasonable to believe that the performance would be significantly improved for more dense D2D network settings. Therefore, this real-world experiment demonstrates the great potential of applying D2D communication and the Lyapunov optimization approach in the design of MCS. More results on the applying backpressure algorithms for opportunistic D2D transmissions can be found in [7], [8], [11].

\section{RAPID, USER-TRANSPARENT, AND SECURE D2D CONNECTIONS}

Since the upper-layer networking algorithms runs on the top of the dynamic D2D network, it is vital to detect and establish the temporary link between opportunistically encountered phones in a rapid and autonomous way, which is also important to fully exploit the potential capacity gain of the short and opportunistic D2D contacts. In addition, the participating mobile users are normally temporarily recruited and are very likely to be strangers, as shown in Fig.5. Therefore, they may not be willing to share data over D2D communications due to security concerns. Given there is no prior trust relations between them, establishing a sharing secret key for the firstly encountered devices (i.e. device pairing) is vital for secure D2D communications.

However, existing device pairing schemes [13], such as WiFi direct pairing secured by the WiFi Protected Setup (WPS) mechanism, require physical interactions (e.g. button clicks) between firstly encountered devices. For instance, each volunteering students in the experiment presented in last section was requested to manually

\footnotetext{
${ }^{3}$ There are many ways to set the sensing rates of smart phones according to specific applications. For instance, the sensing rate of some participating phones can be set as zero, if they are not selected to perform any sensing task (they may still carry out data communication tasks).
} 


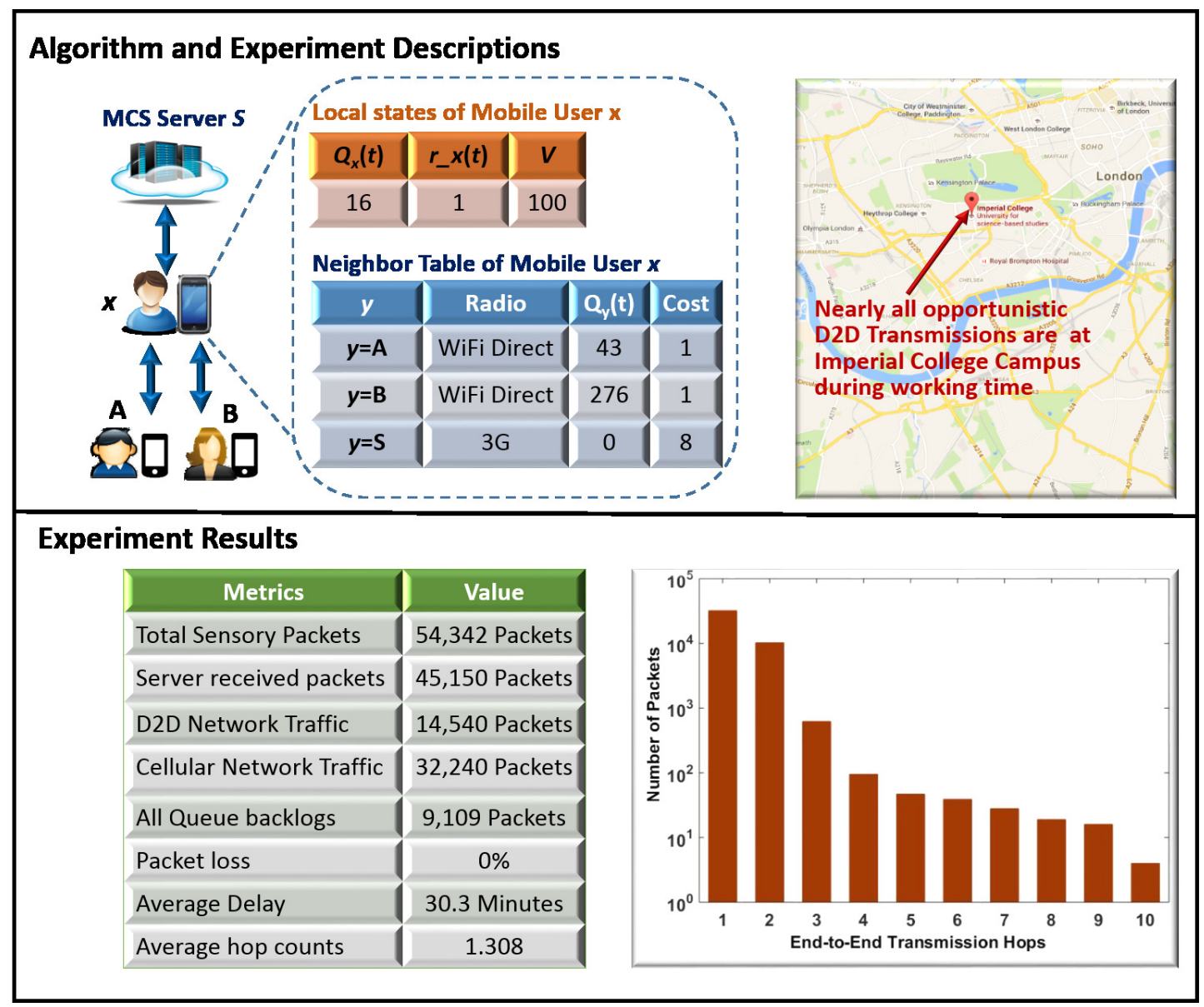

Fig. 4. An three-day experiment to verify the potential of D2D communications and Lyapunov optimization approach in a real-world MCS consisting of a server and 10 Android phones with WiFi direct radios.

authenticate each newly encountered peer device for establishing a WiFi direct connection.

As a result, the lack of efficient D2D connection schemes could be the major obstruction on the road towards the ultimate practical D2D-enabled MCSs. To solve this issue, a new D2D paring scheme, Trustworthy Device Pairing (TDP) is proposed in [14], which manages to work with existing commercial D2D radios and achieves user-transparent and secure D2D connections simultaneously. As shown in Fig.5, TDP has two components: (1) A trust management scheme to profile the trustworthiness of each participating device based on its historical behaviors, and (2) user-transparent D2D paring based on the trust value of each devices.

We have shown that TDP is immune to several potential security threats including the passive eavesdropping and man-in-the-middle attacks, and evaluated its realworld performance in a prototype MCS consisting of a server and two Andorid devices shown in Fig.5. We implemented B-TDP and W-TDP, i.e. TDP with the insecure but user-transparent modes of Bluetooth (JustWork mode) and WiFi direct (blocked WPS) respectively, and compared them with standard Bluetooth (PIN authentication) and the WiFi direct (PBC authentication). The results show that W-TDP achieves much more stable (i.e. much smaller variance in pairing time) and faster D2D initial pairing ${ }^{4}$ than WiFi direct. In addition, B-TDP achieves slightly slower initial pairing but a significantly stability improvement, compared with Bluetooth.

\section{ECONOMIC INCENTIVIZATION FOR SElF-INTEREST AND STRATEGIC MOBILE USERS}

Besides the peer-wise social concerns addressed by device pairing schemes, the network-level social and economic behaviors of the participating "human sensors" needs to be carefully considered in the D2Denabled MCS design. Particularly, the incentivization mechanism, aiming at stimulating the self-interest and strategic mobile users to participate in the system and to follow the operational rules defined by the MCS

\footnotetext{
${ }^{4}$ It is worth noting that initial D2D connection time between two stranger devices is much slower than later ones.
} 


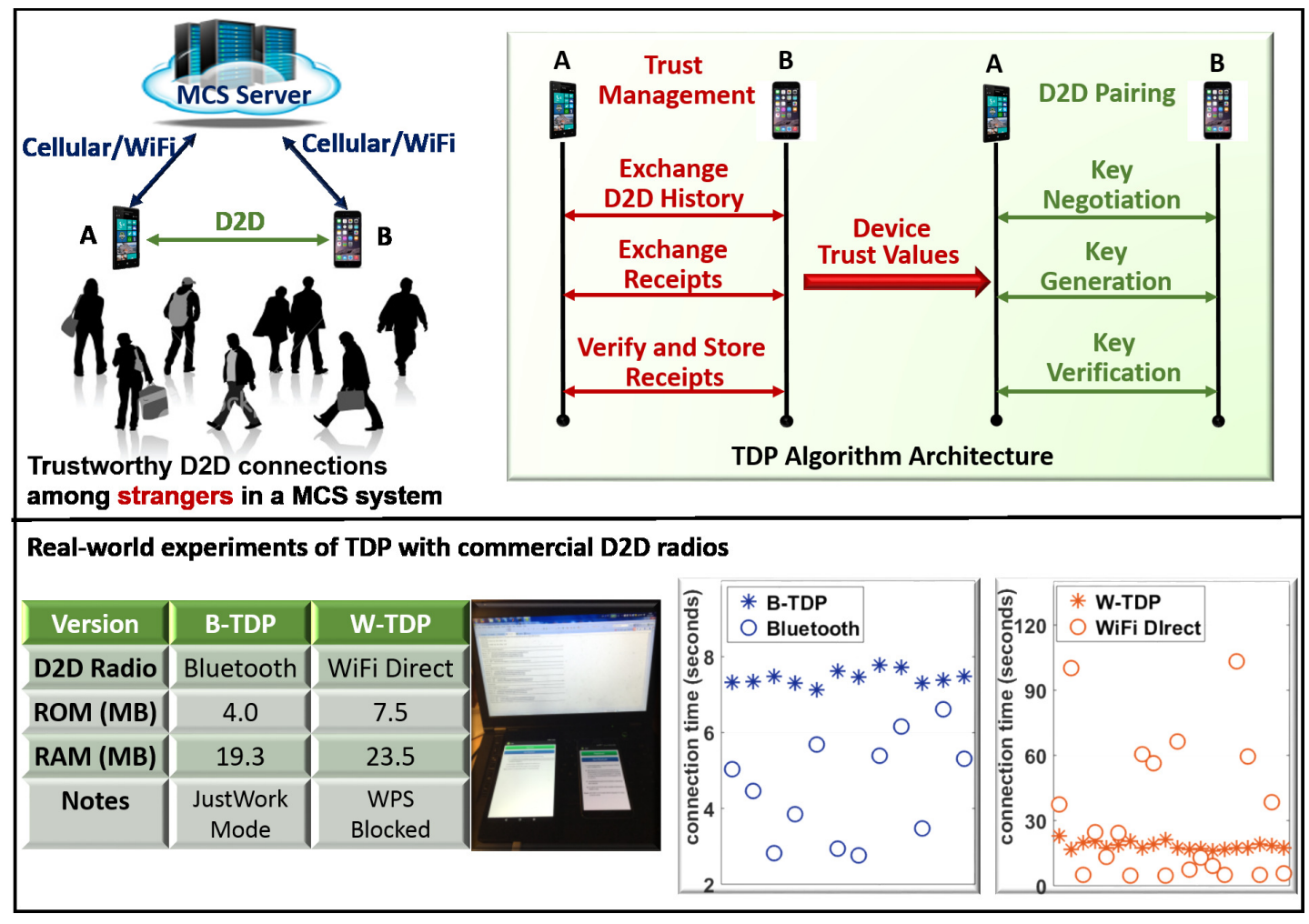

Fig. 5. A D2D pairing algorithm, TDP, for user-transparent and secure opportunistic D2D connections.

system organizers, is vital for the success of a practical MCS implementation [12]. The interactions among the participating individuals significantly complicate the problem space and brings stochastic and unpredictable system dynamics. In the context of D2D-enabled MCSs, an incentivization mechanism is desired to guarantee the following three properties:

- Individual Rationality. As we have discussed, fulfilling MCSs tasks incurs costs in both phone bills and device resource (for sensing, storage, battery, D2D bandwidth, phone bills, etc). In order to motivate a self-interest phone user to participate, a reward that at least covers all costs should be paid by the MCS organizer. In other words, each phone user must be ensured to get some benefits by participating in the system.

- Server Profitability. The MCS organizer (or server) should not incur a deficit, which means a nonnegative profit should be achieved by the MCS organizer.

- Incentive compatibility. A key property of MCS system is that all parameters and operations local to each phone are private and not observable to other phones and the MCS server, as shown in Fig.6(a). As a result, a phone user may try to maximize his or her profit by strategically perform some unfaithful behaviors such as
1) Misreporting Local Parameters (MLP). A participant reports a fake control parameter strategically that can lead wrong system decisions to make benefits. For example, misreporting a fake lower battery level would prevent the system allocating heavy tasks and therefore resulting in longer battery lifetime.

2) Malicious Packet Dropping (MPD). A relaying participant maliciously drops the sensor data of others to reduce the memory and communication cost.

3) Denial of D2D (DoD2D). A participant refuses to communicate with others through D2D radios, especially those have free Internet connections.

Incentive compatibility (or strategy proofness) means that adopting the action suggested by the algorithm intended by the MCS organizer should be the best strategy for each phone user, regardless others actions. This is a strong guarantee to avoid the motivation of cheating behaviors.

\section{A. A Case Study: Combing Lyapunov Optimization and Mechanism Design Theory for the Incentivization of D2D-Enabled MCS}

A recently developed incentivization mechanism, Backpressure meet Taxes (BMT) [7] manages to achieve 


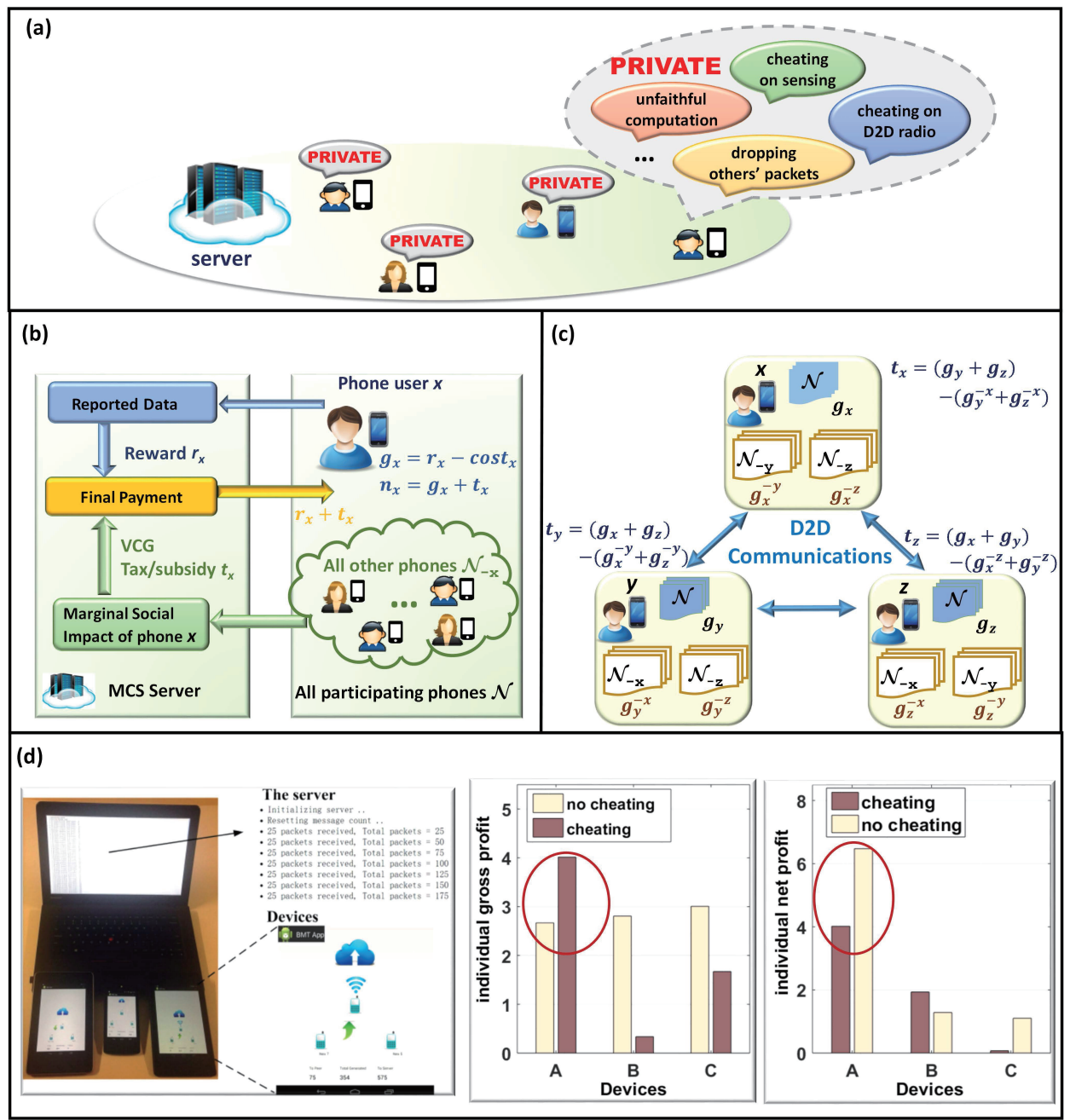

Fig. 6. Potential Cheating behaviors and a distributed incentive compatible algorithm for stochastic D2D-enabled MCS: a) potential unfaithful behaviors in D2D-enabled MCS; b) VCG payment computation for each user $x$; c) distributed VCG tax/subsidy computation; d) a proof-of-concept experiment for BMT.

the three properties for D2D-enabled MCS in a fully distributed way, by combining Lyapunov optimization theory (to address the system dynamics) and the distributed Vickrey-Clarke-Groves (VCG) mechanism design theory (for incentivization). To maximize the social gross profit (i.e. total revenues minus total costs) of the stochastic D2D-enabled MCS, BMT uses a backpressure algorithm, called OptMPSS for distributed sensing and networking over the hybrid D2S and D2D network, which is similar to the simple algorithm discussed in Fig.4.

As shown in Fig.6(b), each phone $x$ will be incurred a monetary cost $\operatorname{cost}_{x}$ for running the OptMPSS. The MCS server will pay each phone user $x$ a reward $r_{x}$ and a monetary transfer $t_{x}\left(t_{x}>0\right.$ means paying a subsidy; $t_{x}<0$ means imposing a tax). With the monetary transfer, each phone user $x$ will aim at maximizing its net profits $\left(n_{x}=r_{x}-\operatorname{cost}_{x}+t_{x}\right)$, rather than its gross profit $\left(g_{x}=r_{x}-\operatorname{cost}_{x}\right)$. In a VCG mechanism, tax/subsidy $t_{x}$ for each phone user $x$ is computed based on $x$ 's contribution of the all other phones in the MCSs (denoted as $\mathcal{N}_{-x}$ ), known as the marginal social impact of $x$. It has been proven in [7] that with VCG tax/subsidy, strictly following all (distributed) operations suggested by OptMPSS is the best strategy of every phone user, regardless other's actions.

However, the key challenge here is to compute the VCG tax/subsidy in a fully distributed manner at realtime. We use an example D2D-enabled MCS with three phones shown in Fig.6(c) to explain how does BMT achieve this. Let us first consider phone $x$, which runs three OptMSS instances: (1) an instance is for real sensing and networking with its D2D neighbors in the real system $\mathcal{N}=\{x, y, z\}$ and records its real gross profit $g_{x}$, (2) two instances for virtual MCS $\mathcal{N}_{-y}=\{x, z\}$ and $\mathcal{N}_{-z}=\{x, y\}$ to compute its virtual gross profit $g_{x}^{-y}$ and $g_{x}^{-z}$ without the presences of $y$ and $z$ respectively, 
i.e. $x$ does not communicate with $y$ or $z$ in $\mathcal{N}_{-y}$ and $\mathcal{N}_{-z}$ respectively, even they have D2D communications in reality. This is also applied for phones $y$ and $z$. In doing so, each phone can compute the contribution of every other phone to itself (e.g. the contribution of phone $y$ to $x$ is $g_{x}-g_{x}^{-y}$ ), and upload this information to the MCS server, who can then compute the VCG tax/subsidy to each phone based on the information collected from all phones. For instance, $x$ 's VCG tax/subsidy allocated by the MCS is $t_{x}=\left(g_{y}+g_{z}\right)-\left(g_{y}^{-x}+g_{z}^{-x}\right)$.

The practical performance of the BMT algorithm was evaluated in a MCS consisting a server and three Android devices A, B, and C, shown in Fig.6(d). Here, device A communicates with MCS server using WiFi (cost 0.1), while both $\mathrm{B}$ and $\mathrm{C}$ use $3 \mathrm{G}$ (cost 1). The three device used WiFi direct for D2D communications with cost 0.1 . It can be seen that in this setting, device A has the motivation of not following the optimal networking actions suggested by BMT, which request A to relay the sensory data traffic of $\mathrm{B}$ and $\mathrm{C}$, resulting in reduction of A's gross profit. Therefore, a natural unfaithful for device A is to disable its WiFi direct, i.e. DoD2D. As shown in Fig.6(d), this cheating behavior indeed contributes to an increase of gross profit but leads to a decrease in net profit, demonstrating the BMT achieves incentive compatibility in practice.

\section{OPEN RESEARCH ISSUES}

The research on exploiting the potential benefits of D2D communications for MCS design is relatively new and many issues have not been addressed. Besides the research topics discussed in this paper, we identify some research opportunities and challenges lie ahead for the design of more efficient D2D-enabled MCS systems.

- Networking for Hybrid Real-Time and DelayTolerant Sensory Data Streams. Since urban sensory data traffics would differ in lifetime. Current approaches in D2D-enabled MCSs are based on opportunistic multi-hop D2D communications, which can only support delay tolerant data traffics. In order to provide strong delay guarantee of real-time sensory data streams, direct cellular and reliable 1hop D2D communications can be used, especially in dense crowd scenarios. Therefore, advanced networking approaches to support the coexisting heterogeneous types of data traffic are highly desired.

- Participator Selection. A key research issue of MCS is to select the best set of participators, according to their sensing quality, locations, and costs. The participator selection problem is widely studied in traditional MCS with pure cellular communications, but all existing approaches in D2D-enabled MCS are restricted by the assumption of a fix set of participators. Therefore, how to jointly optimize and incentive participator selection, sensing, and D2D-based networking is worthy to be systemically investigated.

- Dynamic In-network Processing. D2D communications can enable the opportunistic collaborative interactions among participating phones for the innetwork processing (e.g. data aggregation, fusion, and feature extraction) of the raw urban sensory data streams. Such data pre-processing inside the D2D network would not only significantly decrease the data volume by redundancy reduction, but also improve the efficiency and quality of overall urban big data analytics. Therefore, it is an important but challenging research issue to investigate how to perform and incentive the distributed in-network processing operations for D2D-enabled MCS.

- Exploiting the Fog Architecture. The emerging Fog paradigm [15] has been attracting increasing interests from both academia and industry, due to the low-latency and cost-effective services it can provide at the network edge. Exploiting the hierarchial Fog architecture for D2D-enabled MCS can provide resilient partition of mobile participants across different geographic locations, and adaptive functional partition of different tasks (e.g. sensing, communication, payment, and quality control) over the Cloud server, edge servers, and end devices. This provide great research opportunities to improve the performance in terms of scalability, delay, and bandwidth savings.

\section{CONCLUSION}

This article has provided an overview of recent theoretical and practical developments in Mobile Crowdsensing (MCS) with cooperative Device-to-Device (D2D) communications. We have placed special emphasis on the fundamental challenges for the design of $\mathrm{D} 2 \mathrm{D}$ enabled MCS, covering adaptive sensing and networking, user-transparent and secure D2D connections, and effective incentivization to participating phone users. To further exploit the great potentials of D2D-enabled MCSs, many exciting research opportunities have also been pointed out. We believe that the development of D2D-enabled MCS could not only greatly support urban big data collection, but also provide valuable insights to other systems with social-aware D2D communications, such as D2D-enabled mobile social networking and mobile cloud computing. 


\section{REFERENCES}

[1] R. K. Ganti, F. Ye, and H. Lei, "Mobile crowdsensing: current state and future challenges," IEEE Commun. Mag., vol. 49, no. 11, pp. 32-39, 2011.

[2] B. Guo, Z. Wang, Z. Yu, Y. Wang, N. Y. Yen, R. Huang, and $\mathrm{X}$. Zhou, "Mobile crowd sensing and computing: The review of an emerging human-powered sensing paradigm," ACM Computing Surveys, vol. 48, no. 1, p. 7, 2015.

[3] Y. Zheng, L. Capra, O. Wolfson, and H. Yang, "Urban computing: concepts, methodologies, and applications," ACM Trans. Intelligent Systems and Technology, vol. 5, no. 3, p. 38, 2014.

[4] D. Yang, G. Xue, X. Fang, and J. Tang, "Incentive mechanisms for crowdsensing: Crowdsourcing with smartphones," IEEE/ACM Trans. Network., vol. 24, no. 3, pp. 1732-1744, 2016.

[5] T. Luo, H.-P. Tan, and L. Xia, "Profit-maximizing incentive for participatory sensing," in Proc. IEEE Infocom, 2014.

[6] F. Restuccia and S. K. Das, "Fides: A trust-based framework for secure user incentivization in participatory sensing," in Proc. IEEE WoWMoM, 2014, pp. 1-10.

[7] S. Yang, U. Adeel, and J. McCann, "Backpressure meets taxes: faithful data collection in stochastic mobile phone sensing systems," in Proc. IEEE Infocom, 2015, pp. 1490-1498.

[8] U. Adeel, S. Yang, and J. A. McCann, "Self-optimizing citizencentric mobile urban sensing systems," in Proc. USENIX ICAC, 2014, pp. 161-167.

[9] A. Asadi, Q. Wang, and V. Mancuso, "A survey on device-todevice communication in cellular networks," IEEE Commun. Surveys Tuts., vol. 16, no. 4, pp. 1801-1819, 2014.

[10] D. S. Nunes, P. Zhang, and J. S. Silva, "A survey on human-inthe-loop applications towards an internet of all," IEEE Commun. Surveys Tutor, vol. 17, no. 2, pp. 944-965, 2015.

[11] S. Yang, U. Adeel, and J. A. McCann, "Selfish mules: Social profit maximization in sparse sensornets using rationally-selfish human relays," IEEE J. Sel. Areas Commun., vol. 31, no. 6, pp. 1124-1134, 2013.

[12] F. Restuccia, S. K. Das, and J. Payton, "Incentive mechanisms for participatory sensing: Survey and research challenges," ACM Trans. Sensor Networks, vol. 12, no. 2, p. 13, 2016.

[13] S. Mirzadeh, H. Cruickshank, and R. Tafazolli, "Secure device pairing: A survey," IEEE Commun. Surveys Tuts, vol. 16, no. 1, pp. 17-40, 2014.

[14] C. Zhao, S. Yang, X. Yang, and J. McCann, "Rapid, usertransparent, and trustworthy device pairing for d2d-enabled mobile crowdsourcing," IEEE Trans. Mobile Comput., 2016.

[15] M. Chiang and T. Zhang, "Fog and iot: An overview of research opportunities," IEEE Internet of Things Journal, vol. 3, no. 6, pp. 854-864, 2016.

Shusen Yang [M'13-SM'16] (shusenyang@mail.xjtu.edu.cn) received his Ph.D. degree in Computing from Imperial College London in 2014. He is currently a professor in the National Engineering Laboratory for Big Data Analytics (NEL-BDA) at Xi'an Jiaotong University (XJTU). Before joining XJTU, he worked as a Lecturer (Assistant Professor) at University of Liverpool from 2015 to 2016, and a Research Associate at Intel Collaborative Research Institute (ICRI) for sustainable connected cities from 2013 to 2014. His research interests include Internet of Things (IoTs) and distributed big data analytics. He is a recipient of China "1000 Young Talents Program", an honorary research fellow at Imperial College London, a senior member of IEEE, and a member of ACM and CCF.
Xiaofei Wang [S'06-M'13] (xiaofeiwang@tju.edu.cn) received his B.S. degree from the Department of Computer Science and Technology, Huazhong University of Science and Technology in 2005, and his M.S. and Ph.D. degrees from the School of Computer Science and Engineering, Seoul National University in 2008 and 2013, respectively. He was a Post-Doctoral Research Fellow with the Department of Electrical and Computer Engineering, University of British Columbia. He is currently a Professor with the Tianjin Key Laboratory of Advanced Networking, School of Computer Science and Technology, Tianjin University. His current research interests are social-aware multimedia service in cloud computing, cooperative base station caching, and traffic offloading in mobile content-centric networks.

Usman Adeel (usman.adeel@intel.com) received his B.S. degree from FAST-National University and the MS degree from GIK institute, both in Pakistan. He received the $\mathrm{PhD}$ degree in computing from Imperial College London, United Kingdom, in 2015. He is currently a researcher at Intel Labs Europe. His research interests include in device-to-device communications, mobile crowdsourcing, and opportunistic networking.

Cong Zhao (drzhaocong@gmail.com) received his B.S. and Ph.D. degrees from Xi'an Jiaotong University (XJTU) in 2012 and 2017, respectively. He is currently a Research Associate in the Department of Computing of Imperial College London. His research interests include Edge Computing, Computing Economics, and People-Centric Sensing.

Jia Hu (J.Hu@exeter.ac.uk) is a Lecturer in Computer Science at the University of Exeter. He has published over 40 research papers in prestigious international journals and at reputable international conferences. His research interests include performance evaluation, next generation networking, resource allocation and optimization, energy-efficiency, network security, smart city, and big data.

Xinyu Yang (yxyphd@mail.xjtu.edu.cn) received his B.S., M.S., and $\mathrm{Ph} . \mathrm{D}$. degrees from Xi'an Jiaotong University in 1995, 1997 and 2001, respectively. Currently, he is a professor in the Department of Computer Science and Technology, Xi'an Jiaotong University. His main research interests lie in the areas of wireless networks and network security. He was awarded the title of "New Century Excellent Talent" by the Chinese Ministry of Education in 2009. He is a member of IEEE and ACM.

Julie McCann (j.mccann@imperial.ac.uk) is a Professor in Computer Systems at Imperial College. Her research centers on highly decentralized and self-organizing scalable algorithms for spatial computing systems e.g. wireless sensing networks. She leads both the Adaptive Embedded Systems Engineering Research Group and the Intel Collaborative Research Institute for Sustainable Cities, and is currently working with NEC and others on substantive smart city projects. She has received significant funding though bodies such as the UK's EPSRC, TSB and NERC as well as various international funds, and is an elected peer for the EPSRC. She has actively served on, and chaired, many conference committees and is currently Associative Editor for the ACM Transactions on Autonomous and Adaptive Systems. She is a Fellow of the BCS. 